\title{
Pulmonary interstitial emphysema in very low birthweight infants
}

\author{
S M HART, M MCNAIR, H R GAMSU, AND J F PRICE \\ Departınent of Child Health, King's College Hospital Medical School, London SE5 9RS
}

SUMMARY Forty six of 142 infants weighing less than $1500 \mathrm{~g}$ at birth, who had chest radiographs in the first 5 days of life, developed pulmonary interstitial emphysema (PIE) and in 19 this occurred in the first 24 hours. PIE was seen more frequently in infants weighing less than $1000 \mathrm{~g}$ at birth (24 of 57 ) than in those weighing $1000-1500 \mathrm{~g} \mathrm{(22} \mathrm{of} \mathrm{85).} \mathrm{Ventilation} \mathrm{for} \mathrm{hyaline} \mathrm{membrane} \mathrm{disease} \mathrm{was}$ strongly associated with PIE, and only babies who were resuscitated, or ventilated, or had hyaline membrane disease developed the disorder. Most pneumothoraces were preceded by $x$-ray appearances of PIE (17 of 21). Mortality was increased in ventilated infants who developed PIE and was high in those with severe $x$-ray changes.

Pulmonary interstitial emphysema (PIE) occurs frequently in preterm infants who require mechanical ventilation for severe lung disease. ${ }^{1-4}$ It may impair lung function by reducing compliance, by compressing functional lung tissue, or by restricting pulmonary blood flow. ${ }^{5}$ PIE may also progress to pneumomediastinum and pneumothorax and is associated with an increase in mortality. ${ }^{2}$ As there have been no analyses of PIE in a population of very low birthweight infants we investigated its incidence and its relation to hyaline membrane disease (HMD), mechanical ventilation, resuscitation at birth, pneumothorax, and mortality, in a group of infants who weighed less than $1500 \mathrm{~g}$ at birth.

\section{Patients and methods}

We reviewed retrospectively the notes of all 205 infants of birthweight less than $1500 \mathrm{~g}$ who had been admitted to the neonatal intensive care unit between January 1978 and June 1980. One hundred and fifty seven had radiographic examination of the chest in the first week of life. The radiographs of 5 infants could not be traced. Five infants had congenital abnormalities that could have influenced their clinical course; 1 had hydrocephaly, 1 a cleft palate, 2 had a tracheo-oesophageal fistula, and 1 had congenital emphysema. These infants were excluded from the study. The chest radiographs of the remaining 147 infants were reviewed for HMD, PIE, pneumothorax, and pneumomediastinum by one of us (MM) without knowledge of their clinical course. We graded PIE as follows:
Grade I (Figure (a))-linear translucencies that extended to the lung margin did not taper, and were seen only in the upper zone of the lung.

Grade II (Figure (b)) - more numerous linear translucencies encompassing the mid and lower zones of the lung and a moderate increase in lung volume.

Grade III (Figure (c))-linear and rounded translucencies in all zones of the lung and considerable increase in lung volume.

In 5 infants the radiological appearances were equivocal and it was not possible to say with certainty whether or not PIE was present. It was decided to exclude these babies and confine the analyses to the remaining 142 infants. One hundred and three had radiographs for respiratory distress, 18 for apnoeic attacks (with or without aspiration), 13 for presumed sepsis, and 8 for investigation of a heart murmur. Mean birthweight of these infants was $1041 \mathrm{~g}$ (range $680-1500 \mathrm{~g}$ ) and mean gestational age $28 \cdot 1$ weeks (range 24-34 weeks). Two way contingency tables were used for statistical analyses.

\section{Results}

Forty six of 142 infants $(32 \%)$ had radiological appearances of PIE in the first 5 days of life: in 19 $(13 \%)$ these signs had developed within 24 hours of delivery. PIE was more often seen in infants of birthweight less than $1000 \mathrm{~g}$ than in those above $1000 \mathrm{~g}(\mathrm{P}<0 \cdot 02$; Table $)$.

Radiological signs of HMD were seen in 67 

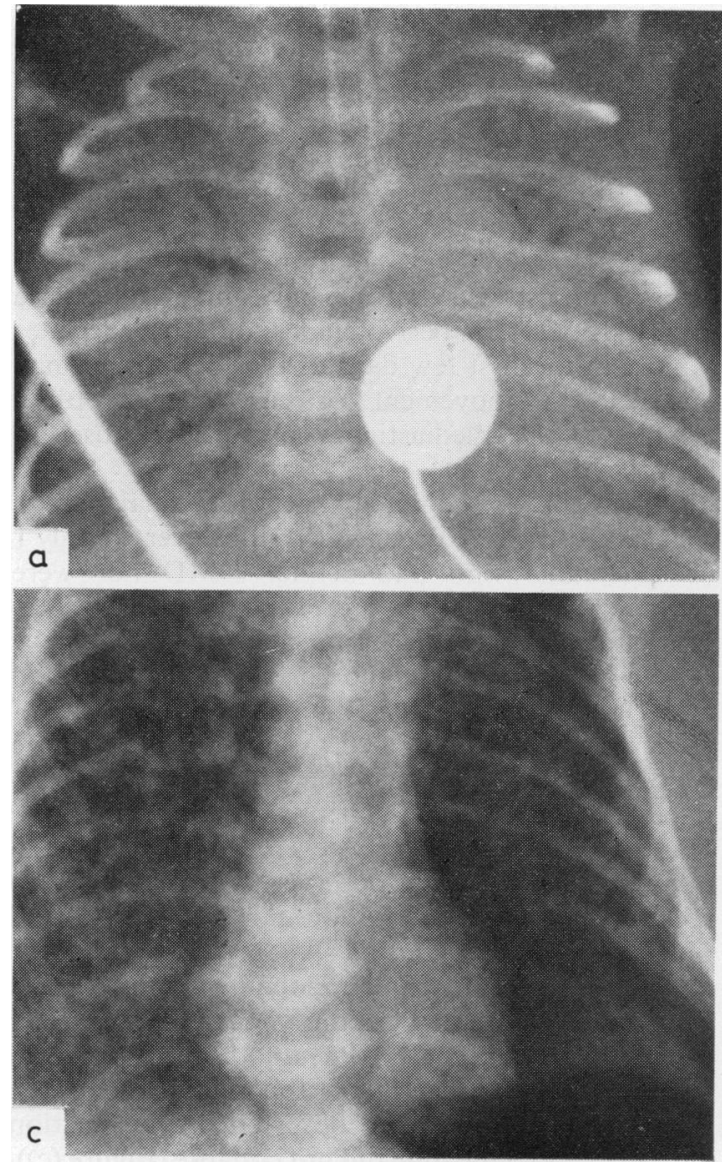

Table Relation between birthweight and pulmonary interstitial emphysema (PIE)

\begin{tabular}{llllll}
\hline Birthweight & $\begin{array}{l}\text { No } \\
\text { PIE }\end{array}$ & \multicolumn{2}{l}{ PIE } & \multicolumn{3}{c}{ Total } \\
\cline { 2 - 5 } & & Grade I & Grade II & Grade III & \\
\hline $\begin{array}{c}<1000 \mathrm{~g} \\
(\mathrm{n}=57)\end{array}$ & 33 & 4 & 10 & 10 & 24 \\
$\begin{array}{c}>1000 \mathrm{~g} \\
(\mathrm{n}=85)\end{array}$ & 63 & 7 & 8 & 7 & 22 \\
\hline Total (n=142) & 96 & 11 & 18 & 17 & 46 \\
\hline
\end{tabular}

infants. An independent review of these babies' notes showed that all had respiratory distress, with grunting, recession, and cyanosis in air within 4 hours of birth and symptoms that persisted for at least 48 hours. Of the infants with HMD, 58 were ventilated. A further 32 infants were ventilated for other reasons.

PIE was associated with HMD and ventilation in 38 of 58 infants $(P<0.0005)$, HMD without ventilation in 2 of 9 infants $(P<0.025)$, and ventilation

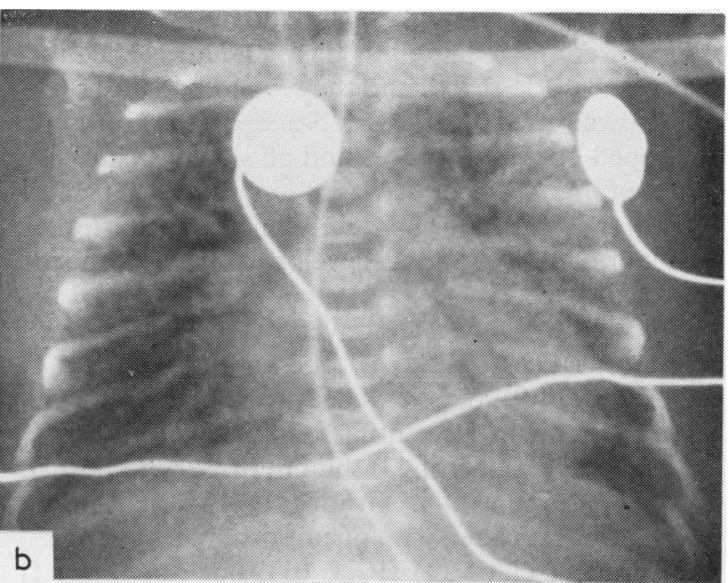

Figure Pulmonary interstitial emphysema in infants of less than $1500 \mathrm{~g}$ birthweight. (a) Grade I.

(b) Grade II. (c) Grade III-there is a left tension pneumothorax but the left lung has not collapsed.

without HMD in 5 of 32 infants $(P<0.05)$. It was more frequently seen in infants with HMD receiving ventilation than in those with HMD who were not ventilated $(P<0.025)$ and those who were ventilated for other reasons $(P<0 \cdot 005)$. Only 1 of 43 infants who were not ventilated and did not have HMD showed radiological signs of PIE.

Ninety three infants were resuscitated at birth with intubation and brief intermittent positive pressure ventilation (less than 10 minutes) using a standard resuscitation apparatus (Resusitaire, Vickers Medical Ltd). The incidence of PIE in infants who were resuscitated but not ventilated ( 2 of 24$)$ was no greater than that in infants who were neither resuscitated nor ventilated ( 1 of $28 ; P>0.5)$. Ventilation was associated with a significant increase in the incidence of PIE both in infants who needed resuscitation at birth $(32$ of $59 ; \mathrm{P}<0.001)$ and in infants who did not (11 of $21 ; \mathbf{P}<0.0005)$.

Three babies who were not ventilated developed PIE. One of these who showed grade I PIE in the first 
24 hours was not resuscitated but did have HMD. The other 2 were resuscitated at birth: 1 had HMD and developed grade II PIE and the second had no HMD but showed grade III PIE. None of these infants developed pneumothoraces and all survived. Twenty seven infants- 9 of birthweight less than $1000 \mathrm{~g}$ and 18 of more than $1000 \mathrm{~g}$-required no resuscitation or ventilation and did not develop HMD. None of these had PIE.

Of the 90 babies who were ventilated, 21 developed pneumothoraces-bilateral pneumothoraces in 6 , right sided in 9, and left sided in 6. Pneumothorax rate was higher in the ventilated infants with previous evidence of PIE (17 of 43) than in those with no PIE (4 of 47) $(P<0.001)$. Grade III PIE was more frequently associated with pneumothoraces (10 of 17) than grade I ( 1 of 11) $(\mathrm{P}<0 \cdot 01)$, but not grade II (6 of 18) $(P>0 \cdot 01)$.

Fifty seven infants died. The overall mortality was significantly greater in babies with PIE (31 of 46) than in those with no signs of PIE (26 of 96) $(P<0.001)$. In babies who were ventilated the mortality was higher in those who had PIE (31 of 43) than in those without PIE (26 of 47) but the difference did not reach statistical significance $(0 \cdot 1>P>0 \cdot 05)$. Among ventilated infants 6 of 10 with grade I PIE, 11 of 17 with grade II PIE, and 14 of 16 with grade III PIE died.

\section{Discussion}

PIE occurs when gas ruptures through the alveoli into the perivascular spaces and becomes trapped in the connective tissue of the peribronchovascular sheaths, the interlobular septa, and the visceral pleura, ${ }^{4}$ and it develops more often if the intrapulmonary connective tissue septa are extensive, ${ }^{6}$ as in the lungs of preterm infants. ${ }^{7}$ Plenat et al. ${ }^{4}$ suggested that PIE increases in frequency with increasing prematurity and in their series 12 of 39 babies with PIE were of less than 32 weeks' gestation. Our data concur with this since PIE was more common in infants of less than $1000 \mathrm{~g}$ birthweight than in infants weighing between 1001 and $1500 \mathrm{~g}$. Severe grades of PIE were slightly though not significantly more frequent in the infants under $1000 \mathrm{~g}$.

We observed PIE in $32 \%$ of 142 very low birthweight infants who required chest radiographs within 5 days of birth. This does not represent the true incidence in our unit, as 48 infants admitted during the period of the study who weighed less than $1500 \mathrm{~g}$ did not require a chest radiograph, and a further 15 infants who had chest radiography were excluded. Given that none of these latter infants developed PIE the incidence is about $22 \%$.
The strong association between PIE and both HMD and mechanical ventilation has been described, ${ }^{2-4}$ and our study supports the view that positive pressure ventilation and reduced lung compliance predispose to its development in preterm infants. In 7 very low birthweight babies we noted severe (grade III) PIE without pneumomediastinum or pneumothorax. Infants with respiratory distress syndrome have an initial increase in interstitial and perivascular fluid that declines rapidly in the first few days of life. ${ }^{8}$ This fluid may obstruct the movement of gas from ruptured alveoli to the mediastinum and thus cause an increase of PIE. ${ }^{2}$

Others have suggested that PIE may occur spontaneously. ${ }^{2}$ We detected PIE, however, in only 1 of 43 infants who had neither HMD nor were ventilated. This infant had received positive pressure ventilation during resuscitation at birth.

About two thirds of the babies studied required resuscitation at birth. Maximum inflation pressure of $25 \mathrm{~cm} \mathrm{H}_{2} \mathrm{O}$ and rates of 20 to 40 breaths a minute were used. We found that infants who were resuscitated but did not subsequently develop HMD or require ventilation rarely developed PIE (1 of 26 infants) and we did not observe PIE in infants who received neither resuscitation nor ventilation and did not develop HMD.

All of the infants who developed pneumothoraces were being ventilated at the time and most had previous evidence of PIE. Pneumothoraces occurred slightly more frequently on the right side. When tension pneumothoraces occurred in association with severe PIE the lung did not collapse (Figure (c)). We therefore considered that it was especially important to take steps to avoid puncturing the lung when inserting chest drains.

The mortality in infants with PIE was high $(67 \%)$. This reflects its association with HMD and the need for ventilation. In ventilated infants, however, we found that the pneumothorax rate and mortality increased with the severity of PIE.

It is difficult to prevent PIE in low birthweight infants with respiratory distress, but it is desirable to avoid overdistension of the lung by high peak inspiratory pressures during resuscitation and ventilation. Pollitzer et al. showed that paralysis with pancuronium of infants requiring ventilation for HMD reduced the duration of oxygen dependence but did not alter the incidence of PIE and pneumothorax. ${ }^{9}$

Several techniques have been described for the management of PIE. Some advocate a conservative approach with regular suction, physiotherapy, and periods of assisted ventilation with high oxygen concentrations. ${ }^{10}$ Others suggest surgical management. 
One infant with unilateral PIE has been treated by lobectomy. ${ }^{11}$ Levine et al. successfully treated 3 of 4 infants with severe PIE in 1 lung by multiple pleurotomy incisions. ${ }^{12}$ If the left lung is predominantly affected it may be possible to manage the infant by selective intubation of the right main bronchus; in 1 report this method was effective in 3 of 4 infants. ${ }^{13}$

High frequency ventilation ( $480-720$ cycles/min) has been used successfully in the management of this disorder. ${ }^{14}$ In addition 2 infants with PIE in whom adequate oxygenation could not be achieved during mechanical ventilation were successfully ventilated by hand at rates of 120 to 140 breaths a minute using maximum inspiratory pressures of $15 \mathrm{~cm}$ $\mathrm{H}_{2} \mathrm{O}$ and expiratory pressures of $3 \mathrm{~cm} \mathrm{H}_{2} \mathrm{O}$. In both infants PIE resolved. ${ }^{15}$ We have had similar success using these rates and pressure settings for mechanical ventilation of 3 infants with severe PIE and think that this approach to the management of PIE in very low birthweight infants deserves further study.

\section{References}

1 Campbell RE. Intrapulmonary interstitial emphysema. A complication of hyaline membrane disease. AJR 1970; 110: 449-56.

2 Thibeault DW, Lachman RS, Laul VR, Kwong MS. Pulmonary interstitial emphysema, pneumomediastinum, and pneumothorax. Occurrence in the newborn infant. Am J Dis Child 1973; 126: 611-4.

3 Burnard ED, Gratton-Smith P, John E. A radiographic, pathologic and clinical study of interstitial emphysema complicating hyaline membrane disease. In: Stern L, Friis-Hansen $\mathrm{B}$, Kildeberg $\mathrm{P}$, eds. Intensive care in the newborn. New York: Masson, 1976: 225-47.
4 Plenat F, Vert P, Didier F, Andre M. Pulmonary interstitial emphysema. Clin Perinatol 1978; 5: 351-75.

5 Macklin CC. Transport of air along sheaths of pulmonic blood vessels from alveoli to mediastinum: clinical implications. Arch Intern Med 1939; 64: 913-26.

6 Reid L. The connective tissue septa in the adult human lung. Thorax 1959; 14: 138-45.

7 Reid L, Rubino M. The connective tissue septa in the fetal human lung. Thorax 1959; 14: 3-13.

8 DeSa DJ. Pulmonary fluid content in infants with respiratory distress. J Pathol 1969; 97: 469-78.

9 Pollitzer MJ, Reynolds EOR, Shaw DG, Thomas RM. Pancuronium during mechanical ventilation speeds recovery of lungs of infants with hyaline membrane disease. Lancet 1981 ; i: 346-8.

10 Leonidas JC, Hall RT, Rhodes OG. Conservative management of unilateral pulmonary interstitial emphysema under tension.J Pediatr 1975; 87: 776-8.

11 Fletcher BD, Outerbridge EW, Youssef S, Bolande RP. Pulmonary interstitial emphysema in a newborn infant treated by lobectomy. Pediatrics $1974 ; 54$ : 808-11

12 Levine D, Trump DS, Waterkotte G. Unilateral pulmonary interstitial emphysema. A surgical approach to treatment. Pediatrics $1981 ; 68: 510-4$.

13 Brooks JG, Bustamante SA, Koops BL, et al. Selective bronchial intubation for the treatment of severe localized pulmonary interstitial emphysema in newborn infants. J Pediatr 1977; 91 : 648-52.

14 Frantz ID, Stark AR, Werthammer J. Improvement in pulmonary interstitial emphysema with high frequency ventilation. Pediatr Res $1981 ; 15$ : 719.

$15 \mathrm{Ng} \mathrm{KPK}$, Easa D. Management of interstitial emphysema by high-frequency low positive pressure hand ventilation in the neonate. J Pediatr 1979; 95: 117-8.

Correspondence to Dr J F Price, Department of Child Health, King's College Hospital Medical School, London SE5 9RS.

Received 13 April 1983 